\title{
Minimum Concentration Normalized by Weight
}

National Cancer Institute

\section{Source}

National Cancer Institute. Minimum Concentration Normalized by Weight. NCI

Thesaurus. Code C92377.

The minimum concentration between dose time and dose time plus Tau (at T min) divided by the weight. 